\title{
Impulsive pinning synchronization of discrete-time network
}

\author{
Zhaoyan $\mathrm{Wu}^{*}$ and Hengjun Wang
}

"Correspondence:

zhywu@jxnu.edu.cn

College of Mathematics and

Information Science, Jiangxi Normal

University, Ziyang Road, Nanchang,

P.R. China

\section{Springer}

\begin{abstract}
Combining impulsive and pinning control, we investigate the synchronization problem of discrete-time network. In the proposed pinning control scheme, the controlled nodes are chosen according to the norm of the synchronization errors at different impulsive instants. Based on the Lyapunov function method and mathematical analysis technique, two synchronization criteria with respect to the impulsive gains and intervals are analytically derived. Both undirected and directed discrete-time networks coupled with Chirikov standard maps are performed in numerical examples to verify the effectiveness of the derived results.
\end{abstract}

Keywords: synchronization; discrete-time network; impulsive control; pinning control

\section{Introduction}

Over the past decade, for better modeling and describing the large-scale physical systems consisting of interactive individuals, many kinds of dynamical networks coupled with continuous- or discrete-time dynamical systems have been introduced [1-26]. For example, discrete-time networks are used to model digitally transmitted signals in a dynamical way [1]. Synchronization, as one of the most important and interesting collective behaviors, has been widely studied, and many valuable results have been obtained. In fact, dynamical networks usually cannot synchronize themselves or synchronize with given orbits without external control. Therefore, many control schemes are proposed to design effective controllers for achieving network synchronization, such as feedback control, intermittent control, impulsive control, pinning control, and so on.

In real world, many complex systems contain large numbers of interactive individuals, that is, the corresponding networks consisting of large numbers of nodes. For this case, applying controllers to all nodes is expensive and even impracticable. Pinning control, as an effective control scheme for reducing the number of controlled nodes, has been extensively used to investigate network synchronization, and many valuable results have been obtained [5-17]. Chen et al. [8] investigated pinning synchronization of complex network using only one controller. Liu et al. [10] studied cluster synchronization in directed networks through combining intermittent with pinning control schemes. Zhang et al. [11] studied pinning control of some typical discrete-time dynamical networks. Mwaffo et al. [12] studied stochastic pinning control of networks of chaotic maps. In [15-17], authors studied synchronization of continuous-time networks through combining impul-

(c) 2016 Wu and Wang. This article is distributed under the terms of the Creative Commons Attribution 4.0 International License (http://creativecommons.org/licenses/by/4.0/), which permits unrestricted use, distribution, and reproduction in any medium, provided you give appropriate credit to the original author(s) and the source, provide a link to the Creative Commons license, and indicate if changes were made. 
sive with pinning control schemes. Wherein, the impulsively controlled nodes are chosen according to the norm of the synchronization errors at distinct control instants. As we know, research on synchronization of continuous- and discrete-time dynamical networks has significant differences. Therefore, extension of the results obtained in [15-17] for continuous-time networks to discrete-time networks is an important issue and deserves further study.

Motivated by the above discussions, in this paper, we investigate the impulsive pinning synchronization of discrete-time dynamical network. At different impulsive instants, the pinned nodes are chosen according to the norm of synchronization errors. Since the synchronization errors are time-varying, the pinned nodes become nonidentical at different impulsive instants. Based on the Lyapunov function method and mathematical analysis approach, we analytically derive two synchronization criteria with respect to the impulsive intervals and gains. The obtained results are verified to be effective and correct by two numerical examples.

The rest of this paper is organized as follows. Section 2 introduces the network model and some preliminaries. Section 3 studies the synchronization of discrete-time network via impulsive pinning control. Section 4 provides two numerical examples to verify the effectiveness of the derived results. Section 5 concludes the paper.

\section{Model and preliminaries}

Consider a discrete-time dynamical network consisting of $N$ nodes described by

$$
x_{i}(k+1)=f\left(x_{i}(k)\right)+\sum_{j=1}^{N} c_{i j} \Gamma x_{j}(k),
$$

where $i=1,2, \ldots, N, x_{i}(k)=\left(x_{i 1}(k), \ldots, x_{i n}(k)\right)^{T} \in R^{n}$ is the state variable of node $i, f: R^{n} \rightarrow$ $R^{n}$ denotes the node dynamics, and $\Gamma=\operatorname{diag}\left\{\gamma_{1}, \gamma_{2}, \ldots, \gamma_{n}\right\} \in R^{n \times n}$ is an inner coupling matrix. The matrix $C=\left(c_{i j}\right)_{N \times N}$ is a zero-row-sum outer coupling matrix, which denotes the network topology and coupling strength and is defined as follows: if there is a connection from node $j$ to node $i(i \neq j)$, then $c_{i j} \neq 0$; otherwise, $c_{i j}=0$.

The objective here is to synchronize network (1) to a given orbit $s(k)$ through designing proper impulsive pinning controllers, where $s(k)$ is a solution of an isolated node satisfying $s(k+1)=f(s(k))$. The controlled network can be written as

$$
\begin{array}{ll}
x_{i}(k+1)=f\left(x_{i}(k)\right)+\sum_{j=1}^{N} c_{i j} \Gamma x_{j}(k), & k=\mathcal{I}_{l-1}+1, \mathcal{I}_{l-1}+2, \ldots, \mathcal{I}_{l}-1, \\
x_{i}(k+1)=x_{i}(k)+U_{i}\left(k, x_{i}(k), s(k)\right), \quad k=\mathcal{I}_{l},
\end{array}
$$

where $l=1,2, \ldots$, the discrete instant set $\left\{\mathcal{I}_{l}\right\}$ satisfies $\mathcal{I}_{l} \in Z^{+}, 0=\mathcal{I}_{0}<\mathcal{I}_{1}<\mathcal{I}_{2}<\cdots<\mathcal{I}_{l}<$ $\cdots$, and $\lim _{l \rightarrow+\infty} \mathcal{I}_{l}=+\infty$, and $U_{i}\left(k, x_{i}(k), s(k)\right)$ are impulsive controllers to be designed.

Let $e_{i}(k)=x_{i}(k)-s(k)$ be the synchronization errors. Choosing the impulsive controllers in $(2)$ as $U_{i}\left(k, x_{i}(k), s(k)\right)=f(s(k))-s(k)+B_{i}(k)\left(x_{i}(k)-s(k)\right)$, we have the following error dynamical system:

$$
\begin{aligned}
& e_{i}(k+1)=f\left(x_{i}(k)\right)-f(s(k))+\sum_{j=1}^{N} c_{i j} \Gamma e_{j}(k), \quad k=\mathcal{I}_{l-1}+1, \mathcal{I}_{l-1}+2, \ldots, \mathcal{I}_{l}-1, \\
& e_{i}(k+1)=e_{i}(k)+B_{i}(k) e_{i}(k), \quad k=\mathcal{I}_{l},
\end{aligned}
$$


where $B_{i}\left(\mathcal{I}_{l}\right) \in R$ are impulsive gains at $k=\mathcal{I}_{l}$, and $B_{i}(k)=0$ for $k \neq \mathcal{I}_{l}, i=1,2, \ldots, N$, $l=1,2,3, \ldots$.

When $k=\mathcal{I}_{l}$, arrange the synchronization errors $e_{i}(k)$ as follows:

$$
\begin{aligned}
\left\|e_{i_{1}(k)}(k)\right\| & \geq\left\|e_{i_{2}(k)}(k)\right\| \geq \cdots \geq\left\|e_{i_{p}(k)}(k)\right\| \\
& \geq\left\|e_{i_{p+1}(k)}(k)\right\| \geq \cdots \geq\left\|e_{i_{N}(k)}(k)\right\|,
\end{aligned}
$$

where $i_{p}(k) \in\{1,2, \ldots, N\}, p=1,2, \ldots, N, i_{p}(k) \neq i_{q}(k)$ for $p \neq q$. Further, if $\left\|e_{i_{p}(k)}(k)\right\|=$ $\left\|e_{i_{p+1}(k)}(k)\right\|$, then let $i_{p}(k)<i_{p+1}(k)$. Let $P\left(\mathcal{I}_{l}\right)=\left\{i_{1}\left(\mathcal{I}_{l}\right), \ldots, i_{p}\left(\mathcal{I}_{l}\right)\right\}$ be a set of $p$ nodes. Choose $B_{i}\left(\mathcal{I}_{l}\right)=b_{l} \in(-2,-1) \cup(-1,0)$ for $i \in P\left(\mathcal{I}_{l}\right)$ and $B_{i}\left(\mathcal{I}_{l}\right)=0$ for $i \notin P\left(\mathcal{I}_{l}\right)$, which means that $P\left(\mathcal{I}_{l}\right)$ is the set of pinned nodes at $k=\mathcal{I}_{l}$.

Assumption 1 The function $f(x)$ satisfies the Lipschitz condition, that is, there exists a positive constant $L$ such that

$$
\|f(y)-f(x)\| \leq L\|y-x\|
$$

for any vectors $x, y \in R^{n}$, where $\|x\|$ denotes the Euclidean vector norm, that is, $\|x\|=$ $\sqrt{x^{T} x}$.

\section{Main result}

In what follows, let $e(k)=\left(e_{1}^{T}(k), e_{2}^{T}(k), \ldots, e_{N}^{T}(k)\right)^{T}, F(e(k))=\left(\left(f\left(x_{1}\right)-f(s)\right)^{T}, \ldots\right.$, $\left.\left(f\left(x_{N}\right)-f(s)\right)^{T}\right)^{T}, \mathcal{T}_{l}=\mathcal{I}_{l}-1-\mathcal{I}_{l-1}$ be the impulsive intervals, $\lambda^{2}$ be the largest eigenvalue of the matrix $\left(C^{T} \otimes \Gamma\right)(C \otimes \Gamma)$ with $\lambda>0, \beta_{l}=\left(1+b_{l}\right)^{2}$, and $\rho_{l}=1-\left(1-\beta_{l}\right) p / N$.

Theorem 1 Suppose that Assumption 1 holds and that there exists a positive constant $\alpha>0$ such that

$$
\ln \rho_{l}+\alpha+2 \mathcal{T}_{l} \ln (L+\lambda)<0, \quad l=1,2, \ldots
$$

Then the synchronization of the discrete-time network (2) with impulsive pinning controllers is achieved.

Proof Consider the following Lyapunov function candidate

$$
V(e(k))=\sum_{i=1}^{N}\left\|e_{i}(k)\right\|^{2} .
$$

When $k=\mathcal{I}_{l-1}+1, \mathcal{I}_{l-1}+2, \ldots, \mathcal{I}_{l}-1$, we have

$$
\begin{aligned}
V(e(k+1)) & =\sum_{i=1}^{N}\left\|e_{i}(k+1)\right\|^{2} \\
& =\|e(k+1)\|^{2} \\
& =\|F(e(k))+C \otimes \Gamma e(k)\|^{2} \\
& \leq(\|F(e(k))\|+\|C \otimes \Gamma e(k)\|)^{2}
\end{aligned}
$$




$$
\begin{aligned}
& \leq(L\|e(k)\|+\lambda\|e(k)\|)^{2} \\
& \leq(L+\lambda)^{2} V(e(k)),
\end{aligned}
$$

which gives

$$
V(e(k+1)) \leq(L+\lambda)^{2\left(k-\mathcal{I}_{l-1}\right)} V\left(e\left(\mathcal{I}_{l-1}+1\right)\right)
$$

When $k=\mathcal{I}_{l}$, we have

$$
\begin{aligned}
V\left(e\left(\mathcal{I}_{l}+1\right)\right) & =\sum_{i=1}^{N}\left\|e_{i}\left(\mathcal{I}_{l}+1\right)\right\|^{2} \\
& =\sum_{i \in P\left(\mathcal{I}_{l}\right)}\left\|e_{i}\left(\mathcal{I}_{l}+1\right)\right\|^{2}+\sum_{i \notin P\left(\mathcal{I}_{l}\right)}\left\|e_{i}\left(\mathcal{I}_{l}+1\right)\right\|^{2} \\
& =\sum_{i \in P\left(\mathcal{I}_{l}\right)}\left(1+b_{l}\right)^{2} \cdot\left\|e_{i}\left(\mathcal{I}_{l}\right)\right\|^{2}+\sum_{i \notin P\left(\mathcal{I}_{l}\right)}\left\|e_{i}\left(\mathcal{I}_{l}\right)\right\|^{2} \\
& =\beta_{l} \sum_{i=1}^{N}\left\|e_{i}\left(\mathcal{I}_{l}^{-}\right)\right\|^{2}+\left(1-\beta_{l}\right) \sum_{i \notin P\left(\mathcal{I}_{l}\right)}\left\|e_{i}\left(\mathcal{I}_{l}^{-}\right)\right\|^{2} .
\end{aligned}
$$

According to the definition of $P\left(\mathcal{I}_{l}\right)$, we have

$$
\frac{1}{N-p} \sum_{i \notin P\left(\mathcal{I}_{l}\right)}\left\|e_{i}\left(\mathcal{I}_{l}\right)\right\|^{2} \leq \frac{1}{N} \sum_{i=1}^{N}\left\|e_{i}\left(\mathcal{I}_{l}\right)\right\|^{2}
$$

Combining (7) and (8), we have

$$
\begin{aligned}
V\left(e\left(\mathcal{I}_{l}+1\right)\right) & \leq\left(\beta_{l}+\frac{\left(1-\beta_{l}\right)(N-p)}{N}\right) \sum_{i=1}^{N}\left\|e_{i}\left(\mathcal{I}_{l}\right)\right\|^{2} \\
& =\rho_{l} V\left(e\left(\mathcal{I}_{l}\right)\right) .
\end{aligned}
$$

For $l=1$, from inequalities (6) and (9) we have

$$
\begin{gathered}
V\left(e\left(\mathcal{I}_{1}\right)\right) \leq(L+\lambda)^{2\left(\mathcal{I}_{1}-1-\mathcal{I}_{0}\right)} V\left(e\left(\mathcal{I}_{0}+1\right)\right) \\
=(L+\lambda)^{2 \mathcal{T}_{1}} V(e(1))
\end{gathered}
$$

For $l=2$, from inequalities (6), (9), and (10) we have

$$
\begin{aligned}
& V\left(e\left(\mathcal{I}_{2}\right)\right) \leq(L+\lambda)^{2\left(\mathcal{I}_{2}-1-\mathcal{I}_{1}\right)} V\left(e\left(\mathcal{I}_{1}+1\right)\right) \\
& =\rho_{1}(L+\lambda)^{2\left(\mathcal{T}_{1}+\mathcal{T}_{2}\right)} V(e(1)), \\
& V\left(e\left(\mathcal{I}_{2}+1\right)\right) \leq \rho_{1} \rho_{2}(L+\lambda)^{2\left(\mathcal{T}_{1}+\mathcal{T}_{2}\right)} V(e(1)) .
\end{aligned}
$$


According to mathematical induction, for any positive integer $l$, we can prove the following inequalities:

$$
\begin{aligned}
& V\left(e\left(\mathcal{I}_{l}\right)\right) \leq \prod_{\sigma=1}^{l-1} \rho_{\sigma} \prod_{\kappa=1}^{l}(L+\lambda)^{2 \mathcal{T}_{\kappa}} V(e(1)), \\
& V\left(e\left(\mathcal{I}_{l}+1\right)\right) \leq \prod_{\kappa=1}^{l}\left(\rho_{\kappa}(L+\lambda)^{2 \mathcal{T}_{\kappa}}\right) V(e(1)) .
\end{aligned}
$$

Assume that inequalities (12) hold for $l \leq m$, Then we have

$$
\begin{aligned}
& V\left(e\left(\mathcal{I}_{m}\right)\right) \leq \prod_{\sigma=1}^{m-1} \rho_{\sigma} \prod_{\kappa=1}^{m}(L+\lambda)^{2 \mathcal{T}_{\kappa}} V(e(1)), \\
& V\left(e\left(\mathcal{I}_{m}+1\right)\right) \leq \prod_{\kappa=1}^{m}\left(\rho_{\kappa}(L+\lambda)^{2 \mathcal{T}_{\kappa}}\right) V(e(1)) .
\end{aligned}
$$

From inequalities (6), (9), and (13) we have

$$
\begin{gathered}
V\left(e\left(\mathcal{I}_{m+1}\right)\right) \leq(L+\lambda)^{2\left(\mathcal{I}_{m+1}-1-\mathcal{I}_{m}\right)} V\left(e\left(\mathcal{I}_{m}+1\right)\right) \\
\leq \prod_{\sigma=1}^{m} \rho_{\sigma} \prod_{\kappa=1}^{m+1}(L+\lambda)^{2 \mathcal{T}_{\kappa}} V(e(1)), \\
V\left(e\left(\mathcal{I}_{m+1}+1\right)\right) \leq \prod_{\kappa=1}^{m+1}\left(\rho_{\kappa}(L+\lambda)^{2 \mathcal{T}_{\kappa}}\right) V(e(1)) .
\end{gathered}
$$

That is to say, inequalities (12) hold for $l=m+1$.

From conditions (5) we have

$$
\rho_{l}(L+\lambda)^{2 \mathcal{T}_{l}}<e^{-\alpha}, \quad l=1,2, \ldots,
$$

and

$$
V\left(e\left(\mathcal{I}_{l}+1\right)\right) \leq e^{-l \alpha} V(e(1))
$$

which implies that $V\left(e\left(\mathcal{I}_{l}+1\right)\right) \rightarrow 0$ as $l \rightarrow \infty$. Then, for $k=\mathcal{I}_{l}+1, \mathcal{I}_{l}+2, \ldots, \mathcal{I}_{l+1}-1$, we have

$$
V(e(k+1)) \leq(L+\lambda)^{2\left(k-\mathcal{I}_{l}\right)} V\left(e\left(\mathcal{I}_{l}+1\right)\right) \rightarrow 0 \quad \text { as } l \rightarrow \infty,
$$

which implies that $\left\|e_{i}(k)\right\| \rightarrow 0$ as $k \rightarrow \infty$, that is, the error system (3) is globally asymptotically stable about zero. Therefore, the synchronization of discrete-time network (2) with impulsive pinning controllers is achieved, and the proof is completed.

If the impulsive gains $b_{l}$ and the impulsive intervals $\mathcal{T}_{l}$ are chosen as a constant $b_{0}$ and a positive constant $\mathcal{T}_{0}$, the following corollary can be easily derived. 
Corollary 1 Suppose that Assumption 1 holds and that there exists a positive constant $\alpha>0$ such that

$$
\ln \rho_{0}+\alpha+2 \mathcal{T}_{0} \ln (L+\lambda)<0
$$

where $\beta_{0}=\left(1+b_{0}\right)^{2}$ and $\rho_{0}=1-\left(1-\beta_{0}\right) p / N$. Then the synchronization of discrete-time network (2) with impulsive pinning controllers can be achieved.

Remark 1 In many existing results about pinning control, the outer coupling matrix is assumed to be irreducible, that is, the network is connected. From the proof of Theorem 1 it is clear that the outer coupling matrix $C$ need not be symmetrical or irreducible. That is, the obtained results can be applied to more general networks and even to disconnected networks.

Remark 2 For any given network, fixing the impulsive gains $b_{l}$ and the number of pinned nodes $p$, we can easily estimate the largest impulsive interval for achieving synchronization from condition (5) or (15).

\section{Numerical simulations}

Consider a discrete-time dynamical network consisting of six nodes and choose the node dynamics as the following Chirikov standard map [12, 27]:

$$
\begin{aligned}
& x_{i 1}(k+1)=x_{i 1}(k)+\eta \sin \left(x_{i 2}(k)\right), \\
& x_{i 2}(k+1)=x_{i 1}(k)+x_{i 2}(k)+\eta \sin \left(x_{i 2}(k)\right),
\end{aligned}
$$

where $x_{i 1}(k)$ and $x_{i 2}(k)$ are taken modulo $2 \pi$ for $i=1,2, \ldots, 6$. This map exhibits chaotic behavior when $\eta>0$. By simple calculations we can choose $L=1+2 \eta$ such that Assumption 1 holds.

Example 1 Consider an undirected discrete-time dynamical network. Choose the inner coupling matrix $\Gamma$ as the identity matrix and the outer coupling matrix as

$$
C=0.005 \times\left(\begin{array}{cccccc}
-5 & 1 & 2 & 1 & 0 & 1 \\
1 & -3 & 0 & 1 & 1 & 0 \\
2 & 0 & -4 & 1 & 0 & 1 \\
1 & 1 & 1 & -5 & 1 & 1 \\
0 & 1 & 0 & 1 & -4 & 2 \\
1 & 0 & 1 & 1 & 2 & -5
\end{array}\right) .
$$

In numerical simulations, choose the number of pinned nodes $p=2$, the impulsive gain $b_{0}=-0.9, \alpha=0.001, \eta=0.01, \mathcal{T}_{0}=3$, and the initial values of $x_{i}(k)$ and $s(k)$ randomly. By simple calculations we have $\lambda=0.0361, \beta_{0}=0.01, \rho_{0}=0.67$, and $\ln 0.67+0.001+$ $6 \ln 1.0561=-0.072<0$, that is, condition (15) in Corollary 1 holds, and the synchronization can be achieved. Figure 1 shows the orbits of the norm of synchronization errors. 


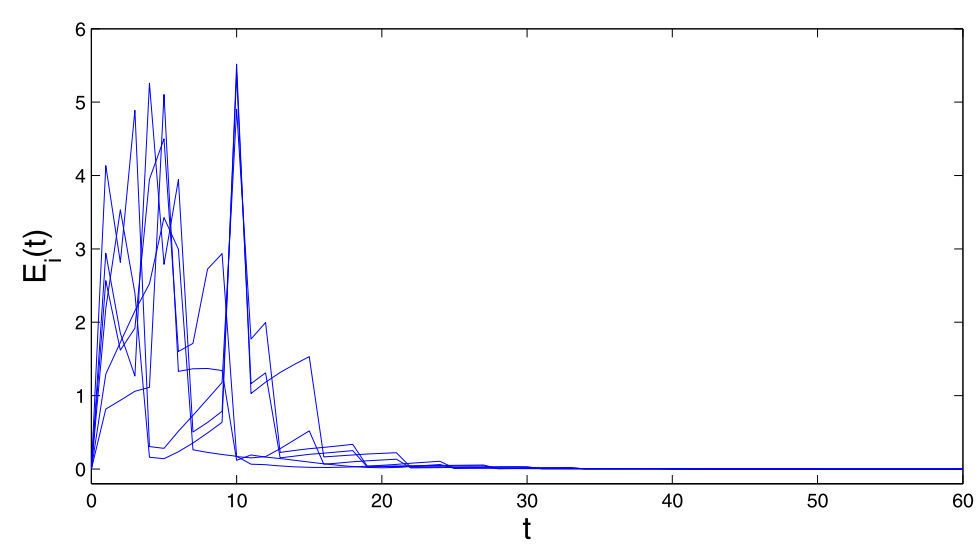

Figure 1 The orbits of the norm of synchronization errors, $E_{i}(t)=\sqrt{e_{i}^{T}(t) e_{i}(t)}, i=1,2, \ldots, 6$.

Example 2 Consider a directed discrete-time dynamical network. Choose the inner coupling matrix $\Gamma$ as identity matrix and the outer coupling matrix as

$$
C=0.005 \times\left(\begin{array}{cccccc}
-5 & 1 & 2 & 1 & 0 & 1 \\
2 & -4 & 0 & 1 & 1 & 0 \\
1 & 1 & -6 & 3 & 0 & 1 \\
0 & 2 & 1 & -5 & 1 & 1 \\
0 & 0 & 2 & 2 & -6 & 2 \\
1 & 1 & 0 & 1 & 1 & -4
\end{array}\right) .
$$

In numerical simulations, choose the number of pinned nodes $p=2$, the impulsive gain $b_{0}=-0.9, \alpha=0.001, \eta=0.01, \mathcal{T}_{0}=3$, and the initial values of $x_{i}(k)$ and $s(k)$ randomly. By simple calculations we have $\lambda=0.0412, \beta_{0}=0.01, \rho_{0}=0.67$, and $\ln 0.67+0.001+$ $6 \ln 1.0612=-0.0431<0$, that is, condition (15) in Corollary 1 holds, and the synchronization can be achieved. Figure 2 shows the orbits of the norm of synchronization errors.

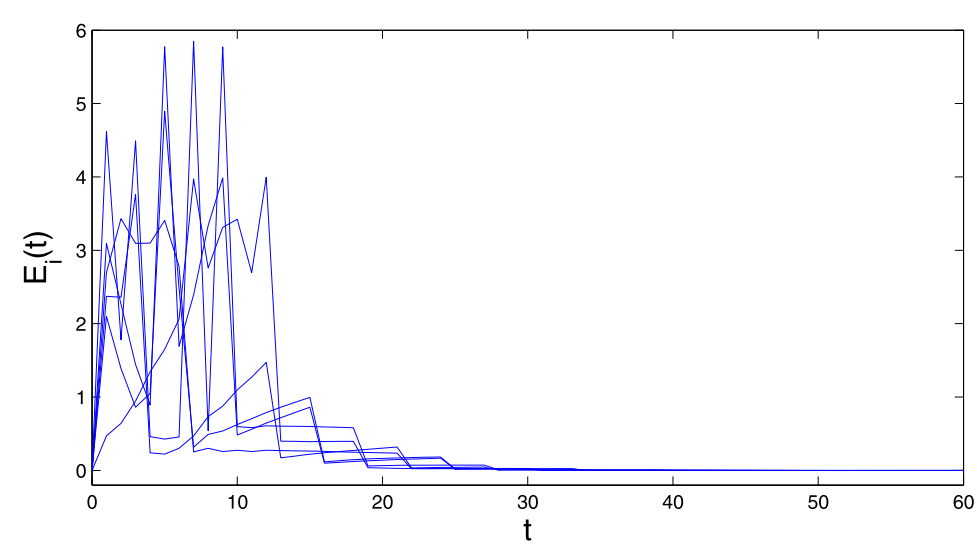

Figure 2 The orbits of the norm of synchronization errors, $E_{i}(t)=\sqrt{e_{i}^{T}(t) e_{i}(t)}, i=1,2, \ldots, 6$. 


\section{Conclusions}

In this paper, we studied the synchronization problem of discrete-time network via impulsive pinning control. In the proposed control scheme, the impulsive controllers are applied to only a fraction of nodes, and the pinned nodes are chosen according to the norm of the synchronization errors at different control instants. Two sufficient conditions for achieving synchronization are derived based on Lyapunov function method and mathematical analysis technique. From the conditions, for any given networks, we can easily estimate the largest impulsive interval by fixing the impulsive gains and the number of pinned nodes $p$. Finally, two numerical examples are performed to illustrate the obtained results.

\section{Competing interests}

The authors declare that they have no competing interests.

\section{Authors' contributions}

All the authors contributed equally to this work. They all read and approved the final version of the manuscript.

\section{Acknowledgements}

This work is jointly supported by the National Natural Science Foundation of China under Grant No. 61463022 and the Natural Science Foundation of Jiangxi Educational Committee under Grant No. GJJ14273.

Received: 8 August 2015 Accepted: 20 January 2016 Published online: 02 February 2016

\section{References}

1. Liang, J, Wang, Z, Liu, Y, Liu, X: Global synchronization control of general delayed discrete-time networks with stochastic coupling and disturbances. IEEE Trans. Syst. Man Cybern., Part B, Cybern. 38, 1073-1083 (2008)

2. Cao, J, Wang, Y: Cluster synchronization in nonlinearly coupled delayed networks of non-identical dynamic systems. Nonlinear Anal., Real World Appl. 14, 842-851 (2013)

3. Cao, J, Alofi, A, Al-Mazrooei, A, Elaiw, A: Synchronization of switched interval networks and applications to chaotic neural networks. Abstr. Appl. Anal. 2013, 940573 (2013)

4. Pan, L, Cao, J, Hu, J: Synchronization for complex networks with Markov switching via matrix measure approach. Appl. Math. Model. 39, 5636-5649 (2015)

5. Wang, X, Chen, G: Pinning control of scale-free dynamical networks. Physica A 310, 521-531 (2002)

6. Li, L, Cao, J: Cluster synchronization in an array of coupled stochastic delayed neural networks via pinning control. Neurocomputing 74, 846-856 (2011)

7. Wang, T, Li, T, Yang, X, Fei, S: Cluster synchronization for delayed Lur'e dynamical networks based on pinning control. Neurocomputing 83, 72-82 (2012)

8. Chen, T, Liu, X, Lu, W: Pinning complex networks by a single controller. IEEE Trans. Circuits Syst. I 54, 1317-1326 (2007)

9. $\mathrm{Ma}, \mathrm{Q}, \mathrm{Lu}, \mathrm{J}$ : Cluster synchronization for directed complex dynamical networks via pinning control. Neurocomputing $101,354-360(2013)$

10. Liu, $X$, Chen, T: Cluster synchronization in directed networks via intermittent pinning control. IEEE Trans. Neural Netw. 22, 1009-1020 (2011)

11. Zhang, H, Li, K, Fu, X: On pinning control of some typical discrete-time dynamical networks. Commun. Nonlinear Sci. Numer. Simul. 15, 182-188 (2010)

12. Mwaffo, V, DeLellis, P, Porfiri, M: Criteria for stochastic pinning control of networks of chaotic maps. Chaos 24, 013101 (2014)

13. Tang, Y, Leung, SYS, Wong, WK, Fang, J: Impulsive pinning synchronization of stochastic discrete-time networks. Neurocomputing 73, 2132-2139 (2010)

14. Deng, L, Wu, Z, Wu, Q: Pinning synchronization of complex network with non-derivative and derivative coupling. Nonlinear Dyn. 73, 775-782 (2013)

15. Lu, J, Kurths, J, Cao, J, Mahdavi, N, Huang, C: Synchronization control for nonlinear stochastic dynamical networks: pinning impulsive strategy. IEEE Trans. Neural Netw. Learn. Syst. 23, 285-292 (2012)

16. Mahdavi, N, Menhaj, MB, Kurths, J, Lu, J, Afshar, A: Pinning impulsive synchronization of complex dynamical networks. Int. J. Bifurc. Chaos 22, 1250239 (2012)

17. Lu, J, Wang, Z, Cao, J, Ho, DWC, Kurths, J: Pinning impulsive stabilization of nonlinear dynamical networks with time-varying delay. Int. J. Bifurc. Chaos 22, 1250176 (2012)

18. Cao, J, Wang, Y, Alofi, A, Al-Mazrooei, A, Elaiw, A: Global stability of an epidemic model with carrier state in heterogeneous networks. IMA J. Appl. Math. 80, 1025-1048 (2015)

19. Zhou, J, Chen, T, Xiang, L: Chaotic lag synchronization of coupled delayed neural networks and its applications in secure communication. Circuits Syst. Signal Process. 27, 833-845 (2005)

20. Luan, $X$, Liu, F, Shi, P: Robust finite-time $H_{\infty}$ control for nonlinear jump systems via neural networks. Circuits Syst. Signal Process. 29, 481-498 (2010)

21. Liu, B, Xia, Y, Mahmoud, MS, Wu, H, Cui, S: New predictive control scheme for networked control systems. Circuits Syst. Signal Process. 31, 945-960 (2012)

22. Li, C, Xu, C, Sun, W, Xu, J, Kurths, J: Outer synchronization of coupled discrete-time networks. Chaos 19, 013106 (2009)

23. Zhang, Q, Lu, J, Zhao, J: Impulsive synchronization of general continuous and discrete-time complex dynamical networks. Commun. Nonlinear Sci. Numer. Simul. 15, 1063-1070 (2010) 
24. Yang, X, Cao, J, Ho, DWC: Exponential synchronization of discontinuous neural networks with time-varying mixed delays via state feedback and impulsive control. Cogn. Neurodyn. 9, 113-128 (2015)

25. Rao, P, Wu, Z, Liu, M: Adaptive projective synchronization of dynamical networks with distributed time delays. Nonlinear Dyn. 67, 1729-1736 (2012)

26. Cao, J, Sivasamy, R, Rakkaiyappan, R: Sampled-data $H_{\infty}$ synchronization of chaotic Lur'e systems with time delay. Circuits Syst. Signal Process. (2015). doi:10.1007/s00034-015-0105-6

27. Chirikov, BV: A universal instability of many-dimensional oscillator systems. Phys. Rep. 52, 263-379 (1979)

Submit your manuscript to a SpringerOpen ${ }^{\circ}$ journal and benefit from:

- Convenient online submission

- Rigorous peer review

- Immediate publication on acceptance

- Open access: articles freely available online

- High visibility within the field

- Retaining the copyright to your article 